\title{
Estresse ocupacional em profissionais das equipes de saúde bucal na estratégia de
}

\section{saúde da família}

\author{
Occupational stress in professionals of the oral health teams in the family health strategy \\ Estrés laboral en los profesionales del equipo de salud bucodental en la estategia de salud de la
}

família

Recebido: 19/03/2021 | Revisado: 26/03/2021 | Aceito: 26/03/2021 | Publicado: 04/04/2021

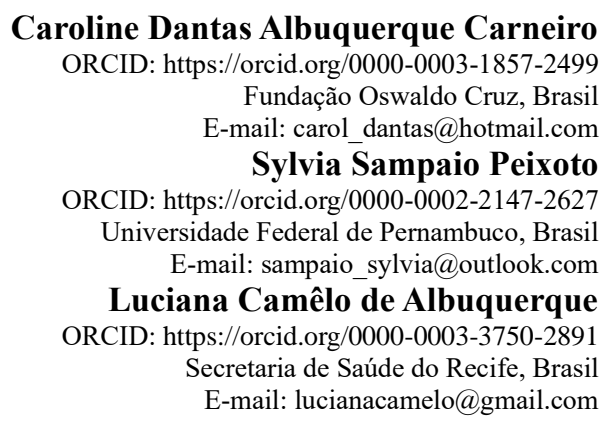

\begin{abstract}
Resumo
Objetivo: O estudo teve por objetivo analisar as condições de estresse ocupacional em que estão submetidos os profissionais de Equipes de Saúde Bucal do Distrito Sanitário II do Recife. Métodos: Estudo descritivo transversal realizado com 66 profissionais da odontologia da Estratégia de Saúde da Família, analisando as situações de esforço e recompensa no trabalho a partir do questionário Effort-Reward Imbalance. Resultados: Segundo o cálculo da razão de desequilíbrio esforço-recompensa, 90,3\% da amostra se encontram em situação de equilíbrio entre esforço despendido no trabalho e recompensa recebida. Porém, $45,5 \%$ encontra-se com alto esforço no trabalho, $53 \%$ com baixa recompensa e $47 \%$ com alto comprometimento excessivo, o que revela uma população que vivencia situações de estresse ocupacional e que está propensa a desordens ao bem-estar no trabalho e na saúde dos profissionais. Conclusão: A adoção de práticas que primem pela igualdade de tratamento e valorização dos profissionais deve ser priorizada, além da legitimação de medidas de vigilância e assistência à saúde dos trabalhadores do município.
\end{abstract}

Palavras-chave: Estresse ocupacional; Odontologia em saúde pública; Atenção primária à saúde; Saúde do trabalhador.

\begin{abstract}
Objective: The study aimed to analyze the occupational stress conditions in which the professionals of Oral Health Teams of the Sanitary District II of Recife are submitted. Methods: Cross-sectional descriptive study conducted with 66 Family Health Strategy dental professionals, analyzing stress and reward situations at work from the Effort-Reward questionnaire. Results: According to the calculation of the effort-reward imbalance ratio, $90.3 \%$ of the sample are in a balance between effort spent at work and reward received. However, $45.5 \%$ have high effort at work, 53\% have low reward and $47 \%$ have high excessive commitment, which reveals a population that experiences situations of occupational stress and is prone to well-being disorders in the workplace. occupational health and health. Conclusion: The adoption of practices that strive for equal treatment and appreciation of professionals should be prioritized, in addition to the legitimation of surveillance and health care measures for workers in the municipality.
\end{abstract}

Keywords: Occupational stress; Public health dentistry; Primary health care; Occupational health.

\section{Resumen}

Objetivo: El estudio tuvo como objetivo analizar las condiciones de estrés laboral en que se encuentran sometidos los profesionales de los Equipos de Salud Bucal del Distrito Sanitario II de Recife. Métodos: Estudio descriptivo transversal realizado con 66 profesionales de la odontología de la Estrategia Salud de la Familia, analizando las situaciones de esfuerzo y recompensa en el trabajo a partir del cuestionario Effort-Reward. Resultados: Según el cálculo de la relación de desequilibrio esfuerzo-recompensa, el 90,3\% de la muestra se encuentra en una situación de equilibrio entre el esfuerzo invertido en el trabajo y la recompensa recibida. Sin embargo, el 45,5\% se encuentran con alto esfuerzo en el trabajo, 53\% con baja recompensa y $47 \%$ con alto compromiso excesivo, lo que revela una población que vive situaciones de estrés laboral y que es propensa a trastornos del bienestar en el trabajo y salud de los profesionales. Conclusión: Se debe priorizar la adopción de prácticas que busquen la igualdad de trato y la valorización de los 
profesionales, además de la legitimidad de las medidas de vigilancia y atención de la salud de los trabajadores del municipio.

Palabras clave: Estrés laboral; Odontologia en salud pública; Atención primaria de salud; Salud laboral.

\section{Introdução}

O trabalho representa um dos fatores determinantes da situação de saúde dos trabalhadores, podendo contribuir para fortalecê-la ou deteriorá-la (Cardoso \& Morgado, 2019). Os sujeitos gastam maior parte do tempo de suas vidas dedicado à atividade laboral, seja dentro ou fora do ambiente e expediente de trabalho. Logo, o trabalho deveria significar realização, satisfação e prazer (do Prado Tostes, 2017). Entretanto, especialmente na sociedade capitalista, o trabalho se apresenta como um meio de exploração e desigualdade social, e, consequentemente, atua como fonte ou intensificador de problemas de saúde (Iora, 2020).

As doenças profissionais e as relacionadas ao trabalho são responsáveis por grande parte do absenteísmo, da incapacidade temporária ou permanente e até de invalidez para o trabalho, acarretando grande custo econômico para os sistemas de seguridade social (Altafim, 2018). Entre os anos de 2012 e 2016, o pagamento, pelo Instituto Nacional de Seguridade Social (INSS), de benefícios concedidos por incapacidade temporária, os auxílios-doença, além de aposentadorias por invalidez foi um valor de R\$ 81.358.732.105,65 (Brasil, 2017).

Atualmente, uma das principais causas de adoecimento é o estresse ocupacional, instituindo-se como importante fator de risco ao bem-estar psicossocial do indivíduo, afetando diretamente a saúde e a qualidade de vida afetiva, social e profissional (Zenkner, Denardin, Jesus, Strom, Silva \& Carlesso, 2020). O estresse ocupacional é considerado como a soma de cargas físicas, mentais e fisiológicas que, quando se tornam intensas, a ponto de ultrapassar a capacidade de enfrentamento do indivíduo, se convertem em reações emocionais negativas (do Prado, 2016).

Estudos apontam uma série de manifestações comuns aos profissionais de saúde quando expostos ao sofrimento imposto pelas relações, volume e condições de trabalho, sendo algumas delas: tensão muscular, insegurança, desânimo, isolamento, distúrbios gástricos, aumento na ingestão de substâncias psicoativas, irritabilidade, distanciamento físico (absenteísmo) ou mental (falta de atenção), depressão, medo, ansiedade e suicídio (Maia, 2016).

Os profissionais de odontologia estão entre os que trabalham sob maior nível de estresse, podendo estar relacionado a estressores como pressões econômicas, pressão com o tempo, trabalho com pacientes ansiosos e pouco cooperativos, infringir dor aos pacientes, limitações do campo visual, ruídos, radiação, efeitos do trabalho prolongado em determinada posição física, repetição do trabalho, confinamento no consultório, dentre outros (dos Santos et al., 2016).

Além dos fatores já citados, tem-se as especificidades do trabalho na Estratégia de Saúde da Família (ESF), onde os profissionais estão em contato diário com a realidade de comunidades que, em sua grande parcela, são carentes em diversos aspectos (Altafim, 2018). Apesar de se depararem com complexas demandas, os recursos são escassos para atendê-las, além de, muitas vezes, ter que conviver com ambientes insalubres, inseguros e propícios a riscos à saúde, situações que atuam como fatores estressores na vida dos trabalhadores, somadas às exigências e o não reconhecimento do trabalho (de Oliveira \& de Araujo, 2018).

No Brasil, a maioria dos estudos relacionados ao estresse ocupacional e suas consequências são realizados em instituições privadas, pela diminuição de produção, afastamentos e outros prejuízos ao trabalho. Logo, achou-se pertinente realizar estudo com o objetivo de analisar as condições de estresse ocupacional em que estão submetidos os profissionais de Equipes de Saúde Bucal (ESB) na ESF. 


\section{Procedimentos Metodológicos}

Trata-se de um estudo exploratório-descritivo de corte transversal com abordagem quantitativa e qualitativa, realizado com os profissionais das 32 ESB das Unidades de Saúde da Família (USF) do Distrito Sanitário II da cidade do Recife-PE.

Participaram da pesquisa cirurgiões-dentistas (CD), técnicos de saúde bucal (TSB) e auxiliares em saúde bucal (ASB) que atuam na ESF. Os critérios de exclusão foram os afastamentos, incluindo férias e licença, que ocorreram no período da coleta de dados, que se deu de março a maio de 2018.

Para responder ao questionário, os profissionais foram contatados no próprio local de trabalho, onde a pesquisadora responsável os apresentava o Termo de Consentimento Livre e Esclarecido (TCLE) e coletava a assinatura dos participantes.

Aplicou-se um questionário estruturado, contendo questões sobre informações pessoais, econômicas e sociodemográficas. Além disso, avaliou-se o estresse ocupacional a partir do questionário Effort Reward Imbalance (ERI). Segundo Schnall, Landsbergis e Baker (1994), este questionário elaborado por Johannes Siegrist de "esforço-recompensa” é o modelo mais abrangente de abordar o estresse no trabalho. Segundo os autores, uma vantagem do modelo é a inclusão da segurança no trabalho e a mobilidade ascendente (perspectivas de promoção).

O modelo prever que o esforço no trabalho faz parte do contrato social, mas que demanda de uma adequada recompensa, sendo esta por meio de dinheiro, estima, oportunidades na carreira e segurança no emprego. Estes elementos são influentes na saúde do trabalhador, e seu desequilíbrio podem gerar reações fisiológicas e emocionais negativas. Então, levando em conta tais pressupostos, o ERI avalia as fontes geradoras de estresse no ambiente laboral e na saúde do trabalhador, com ênfase nas situações em que não há a reciprocidade entre esforço e recompensa (Almeida, 2016).

Na primeira etapa do questionário, foram coletadas informações sobre as variáveis: profissão, idade, sexo, situação conjugal, filhos, bairro em que reside, meio de transporte utilizado para ir ao trabalho, tempo médio gasto para chegar ao trabalho, escolaridade, renda média mensal, tempo de trabalho na área, total de horas dedicadas ao trabalho semanalmente e motivos que já os levaram a se afastar do trabalho.

O estresse ocupacional foi avaliado de acordo com os pressupostos do ERI. Este questionário contém 23 questões e é dividido em três dimensões: esforço no trabalho (6 itens), recompensa (11 itens) e comprometimento excessivo com o trabalho (6 itens).

Na dimensão de esforço, o questionário investiga a ocorrência de situações incômodas na rotina de trabalho que venham a causar algum nível de estresse, como, por exemplo, pressão do tempo, interrupções, esforço físico e altos níveis de exigência.

$\mathrm{Na}$ segunda, o instrumento aborda questões de recompensa que o trabalho pode oferecer, no sentido financeiro, da estima (por exemplo, respeito e apoio dos colegas de trabalho e de seus chefes) e do status no trabalho (a exemplo da estabilidade, chances de promoção e reconhecimento).

As questões da dimensão de comprometimento excessivo buscam investigar o envolvimento do trabalhador para além do ambiente ocupacional, através de reações como a sensação de estar "sufocado" pela pressão do tempo, não conseguir "se desligar" facilmente do trabalho, não ter sono tranquilo devido a problemas no trabalho, entre outras.

Nas duas primeiras dimensões, o participante, inicialmente, informa se concorda ou não com o enunciado da questão e, em seguida, deve escolher o nível de estresse que ele vivencia na situação relatada, podendo variar entre: (1) nem um pouco estressado; (2) um pouco estressado; (3) estressado; (4) muito estressado. Na dimensão de comprometimento excessivo, as opções de respostas são: (1) discordo totalmente; (2) discordo; (3) concordo; (4) concordo totalmente.

Para a análise, houve a dicotomização das escalas esforço, recompensa e comprometimento excessivo em alto e baixo. Para isto, foi feito o somatório das questões referentes a cada escala e utilizou-se a mediana como ponto de corte. O cálculo da razão de desequilíbrio esforço-recompensa é feito por meio da equação: $(\Sigma \mathrm{E} \div[\Sigma \mathrm{R} \times \mathrm{c}])$, onde $\mathrm{E}=$ esforço extrínseco, $\mathrm{R}=$ 
recompensa, multiplicada pelo fator de correção $(c=0,545455)$. O modelo ERI prediz que resultados inferiores a 1 indicam situação de equilíbrio entre esforços e recompensa, e superiores a 1 indicam situação de desequilíbrio entre esforço despendido e recompensa recebida no trabalho (Almeida, 2016).

A análise foi do tipo descritiva, abordando inicialmente a caracterização da população, incluindo as variáveis sobre idade, sexo, tempo de trabalho, renda mensal, dentre outras. Já a descrição dos fatores estressores ocorreu de acordo com o modelo ERI, analisando os escores de cada uma das escalas e também da razão entre esforço-recompensa.

A pesquisa foi realizada de acordo com os preceitos da Resolução do Conselho Nacional de Saúde/CNS número 466/12, a qual estabelece diretrizes e normas reguladoras de pesquisas envolvendo seres humanos. A coleta de dados só foi realizada após aprovação no Comitê de Ética e Pesquisa do Hospital Agamenon Magalhães (Pernambuco), com autorização sob o CAAE de número 84923718.5.0000.5197.

\section{Resultados e Discussão}

Dos 69 profissionais de saúde bucal lotados em 32 das USF do Distrito Sanitário II do Recife, 66 participaram da pesquisa, respondendo ao questionário. A distribuição da amostra, segundo categoria profissional, se deu da seguinte forma: 31 CD, 26 ASB e 09 TSB. Essas e outras características podem ser encontradas na Tabela 1.

Tabela 1. Características sociodemográficas e econômica dos profissionais de saúde bucal da Estratégia de Saúde da Família do Distrito Sanitário II, Recife.

\begin{tabular}{|c|c|c|}
\hline Variáveis & $\mathbf{N}$ & $\%$ \\
\hline \multicolumn{3}{|l|}{ Profissão } \\
\hline Cirurgiã(o)-dentista & 31 & 47,0 \\
\hline Técnico em Saúde Bucal & 09 & 13,6 \\
\hline Auxiliar de Saúde Bucal & 26 & 39,4 \\
\hline \multicolumn{3}{|l|}{ Idade } \\
\hline Menos de 30 anos & 03 & 4,5 \\
\hline 30 a 39 anos & 26 & 39,4 \\
\hline 40 a 49 anos & 25 & 37,9 \\
\hline 50 a 59 anos & 09 & 13,6 \\
\hline 60 anos ou mais & 03 & 4,5 \\
\hline \multicolumn{3}{|l|}{ Sexo } \\
\hline Masculino & 07 & 10,6 \\
\hline Feminino & 59 & 89,4 \\
\hline \multicolumn{3}{|c|}{ Escolaridade por categoria } \\
\hline \multicolumn{3}{|l|}{$\underline{\text { Cirurgiões-dentistas }}$} \\
\hline Ensino superior & 01 & 3,2 \\
\hline Especialização & 28 & 90,3 \\
\hline Doutorado & 02 & 6,4 \\
\hline \multicolumn{3}{|l|}{ Técnicos de Saúde Bucal } \\
\hline Ensino médio completo & 01 & 11,1 \\
\hline Ensino técnico & 06 & 66,6 \\
\hline Ensino superior & 01 & 11,1 \\
\hline Especialização & 01 & 11,1 \\
\hline
\end{tabular}




\begin{tabular}{|c|c|c|}
\hline \multicolumn{3}{|l|}{$\underline{\text { Auxiliares em Saúde Bucal }}$} \\
\hline Ensino médio completo & 07 & 26,9 \\
\hline Ensino técnico & 07 & 26,9 \\
\hline Ensino superior & 11 & 42,3 \\
\hline Especialização & 01 & 3,8 \\
\hline \multicolumn{3}{|l|}{ Renda salarial total mensal } \\
\hline 1 a 3 salários mínimos & 32 & 48,5 \\
\hline 4 a 6 salários mínimos & 08 & 12,1 \\
\hline 7 a 9 salários mínimos & 24 & 36,4 \\
\hline Mais de 10 salários mínimos & 02 & 3,0 \\
\hline \multicolumn{3}{|l|}{ Tempo de trabalho na área } \\
\hline Até 5 anos & 05 & 7,6 \\
\hline 6 a 10 anos & 15 & 22,7 \\
\hline 11 a 15 anos & 23 & 34,8 \\
\hline 16 a 20 anos & 11 & 16,7 \\
\hline 21 a 25 anos & 12 & 18,2 \\
\hline \multirow{2}{*}{\multicolumn{3}{|c|}{$\begin{array}{l}\text { Total de horas dedicadas ao } \\
\text { trabalho semanalmente }\end{array}$}} \\
\hline & & \\
\hline Até 40 horas & 47 & 71,2 \\
\hline$>40$ até 60 horas & 12 & 18,2 \\
\hline$>60$ até 80 horas & 7 & 10,6 \\
\hline
\end{tabular}

Fonte: Autores.

Considerando os aspectos sociodemográficos, 89,4\% $(n=59)$ da amostra foi composta por mulheres. A feminização no setor saúde é uma tendência mundial e as profissões que eram tradicionalmente masculinas, como medicina e odontologia, estão com taxas cada vez mais elevadas de participação feminina (de Brito, Mendes, dos Santos Neto \& de Farias, 2016).

Apenas 4,5\% $(n=03)$ da população tem idade abaixo dos 30 anos e 69,7\% ( $\mathrm{n}=46)$ possui mais de 10 anos de carreira de trabalho na odontologia, revelando um grupo de profissionais mais maduro e experiente. Em consonância com o cenário mundial, o Brasil experimenta um quadro de envelhecimento da população e, consequentemente, da classe trabalhadora, devendo-se haver cada vez mais vigilância dessas atividades laborais e adequação delas para receber e colaborar com o envelhecimento ativo (Costa \& Soares, 2018).

Dos participantes, 19 profissionais $(28,8 \%)$ possuem outro vínculo empregatício, chegando a trabalhar até 80 horas por semana. A ocorrência de múltiplos vínculos empregatícios é uma realidade dentre os profissionais da área de saúde, sob a justificativa de receberem baixa remuneração em seus postos de trabalho. Temos, por consequência, prejuízos na qualidade da assistência prestada e danos à saúde física e mental dos profissionais (Altafim, 2018).

Analisando os resultados obtidos do questionário ERI, observou-se que 45,5\% (n=30) da população mostra-se com índice de alto esforço no trabalho. Nesta dimensão, 37,9\% $(\mathrm{n}=25)$ se sentem pressionados pelo tempo, relatando ficar, em sua maioria, um pouco estressado $(n=15)$ e estressado $(n=09)$ com esta situação; 43,9\% $(n=29)$ relatam ser frequentemente interrompidos e incomodados no trabalho e, com isso grande parte fica um pouco estressados $(\mathrm{n}=18)$ e estressados $(\mathrm{n}=07)$ e $86,4 \%(n=57)$ dos participantes concordam que possuem muita responsabilidade em seu trabalho e, para a maioria desses, isso não causa nenhum estresse $(n=19)$ ou um pouco de estresse $(30)$.

Estudos revelam que o esforço físico na rotina de trabalho da odontologia pode gerar distúrbios osteomusculares 
relacionados ao trabalho que, por sua vez, podem levar os profissionais a se afastarem temporária ou definitivamente do trabalho (Oliveira, de Braz, Urbano, dos Santos \& Araki, 2018). No presente estudo, 53\% ( $\mathrm{n}=35)$ dos profissionais concordam que seu trabalho exige muito esforço físico, o que gera um pouco de estresse na maioria $(\mathrm{n}=21)$ deles.

Dentre os profissionais de saúde bucal participantes, 44\% ( $\mathrm{n}=29)$ concordam que nos últimos anos, o trabalho passou a exigir cada vez mais deles, o que gera, em sua maioria, um pouco de estresse $(n=21)$. Porém os $56,1 \%(n=37)$ que discordam da afirmativa estão em consonância com a literatura que diz que com o passar dos anos, os profissionais tendem a se adaptarem às condições difíceis da atividade laboral, aprendendo a criar estratégias para protege-los dessas situações (Marcelino Filho \& Araújo, 2015).

De acordo com as questões da dimensão recompensa, a relação interpessoal entre os participantes e seus colegas de trabalho e entre eles e seus chefes não geram, na maioria dos casos, nenhum nível de estresse. Quando questionados sobre receber respeito dos chefes, dos colegas de trabalho e contar com apoio em situações difíceis, em média 84,3\% ( $\mathrm{n}=56$ ) concordaram com as afirmativas. Estudos mostram que o bom relacionamento com os colegas no ambiente trabalho é uma importante questão para a harmonia e o bem-estar dos profissionais, trabalhando como fator de proteção ao estresse ocupacional (Hirschle \& Gondim, 2020).

Ao analisar os resultados das três dimensões do ERI, observou-se uma distribuição equilibrada entre elas, sendo: 45,5\% $(n=30)$ da amostra com alto esforço no trabalho, 53\% $(n=35)$ com baixa recompensa e $47 \%(n=31)$ com alto comprometimento excessivo (Tabela 2), o que revela uma vivência dos trabalhadores a situações de estresse ocupacional, predispondo desordens ao bem-estar no trabalho e na saúde dos profissionais como um todo.

Tabela 2. Frequência de esforço e recompensa pelos profissionais de saúde bucal da Estratégia de Saúde da Família do Distrito Sanitário II, Recife.

\section{Modelo esforço-recompensa}

Esforço (mediana $=5)$

Baixo

Alto

Recompensa (mediana $=9)$

Baixa

Alta

Comprometimento excessivo (mediana $=13)$

Baixo

Alto
$\mathbf{N}$ $\%$ 36

35

31

Fonte: Autores.

Entre os que obtiveram alta recompensa, 61,3\% $(\mathrm{n}=19)$ eram $\mathrm{CD}$, dado que corrobora com os achados de Marcelino Filho e Araújo (2015), que evidenciam que esses profissionais apresentaram a maior frequência (50\%) de alta recompensa dentre as categorias de saúde pesquisadas.

$77,7 \%$ dos TSB $(n=07)$ e $61,5 \%$ dos ASB $(n=16)$ se enquadraram no estado de baixa recompensa, alertando sobre prováveis situações de desigualdade e tratamento diferenciado dado às categorias profissionais, principalmente entre as de nível 
superior e a equipe auxiliar.

Ainda em relação às diferenças entre as categorias, no que concerne aos aspectos econômicos, todos os 32 participantes que relataram receber como renda mensal de um a três salários mínimos são técnicos e auxiliares de saúde bucal. Desses, 62,5\% $(\mathrm{n}=20)$ relatam ficar estressados ou muito estressados com a situação salarial. Em análise aos vencimentos de cada uma das categorias, observamos que o salário base da equipe auxiliar no período pesquisado é praticamente o mesmo: $\mathrm{R} \$ 1.358,02$ para técnicos e R \$1.367,00 para auxiliares. Quando comparados com o salário base da categoria de CD, observa-se que estes chegam a receber mais de quatro vezes o valor do salário dos demais profissionais da ESB ( $\$$ \$ 5.773,29). Tais fatos colaboram para o mal-estar e a desarmonia entre os profissionais das ESB, podendo acarretar repercussões negativas até para o estado de saúde dos profissionais envolvidos.

A razão entre esforço-recompensa da amostra pesquisada revelou que 90,3\% $(n=56)$ dos profissionais estão em estado de equilíbrio entre esforço despendido e recompensa recebida. Os resultados positivos são dados relevantes no que concerne à gestão do trabalho do município, visto que o modelo ERI prediz que a falta de reciprocidade entre esforço e recompensa pode gerar experiencias de estresse, havendo consequências para a saúde física e mental do trabalhador (Siegrist et al., 2004; Chor, Werneck, Faerstein, Alves \& Rotenberg, 2008). Porém, os dados devem ser analisados com cautela, pois os mesmos podem ter sofrido influência de vieses, sendo alguns deles analisados a seguir.

O vínculo estável é uma realidade dos profissionais participantes, visto que todos são estatutários, e 95,5\% (n= 63) da amostra concorda que possuem estabilidade no emprego. Este fato pode funcionar como fator de proteção a situações de estresse, devido à seguridade de um vínculo por tempo indeterminado, proteção social trabalhista e previdenciária, direito a greves, negociações, dentre outras vantagens em relação aos vínculos precários e instáveis.

O fato de 95,5\% ( $\mathrm{n}=63)$ dos participantes ter idade acima dos 30 anos é um fator que colabora com o bem-estar e equilíbrio no ambiente laboral. Trindade e Lautert (2010), em pesquisa com profissionais da Estratégia de Saúde da Família, encontrou maior prevalência da síndrome de burnout em profissionais jovens, principalmente nos que ainda não alcançaram os 30 anos. Além da falta de confiança em assumir as responsabilidades do trabalho, jovens idealizam mais e ficam mais propensos a desilusões (Trindade \& Lautert, 2010).

Dentre a população participante da pesquisa, $69,7 \%(\mathrm{n}=46)$ já tem mais de 10 anos de carreira profissional na odontologia. Em estudo realizado por Lima, Farah e Bustamante-Teixeira (2018), que buscou identificar a prevalência de burnout nos profissionais da atenção primária, foi encontrada uma menor prevalência nos profissionais com mais de 10 anos de serviço, demonstrando que profissionais mais experientes adquirirem confiança e maior habilidade em lidar com fatores estressores no ambiente de trabalho.

Devemos considerar também que esse tipo de pesquisa revela o estado na qual o participante se encontra no momento da aplicação do instrumento. Portanto, os resultados podem não expor realmente as vivências de estresse ocupacional na rotina das categorias participantes. Por fim, devemos considerar algum suposto medo dos participantes de que, baseado nas respostas dadas, pudessem ocorrer punições aos mesmos, caso não fosse mantida a confidencialidade da pesquisa.

\section{Conclusão}

A partir dos resultados obtidos, podemos concluir que, a partir do cálculo da razão entre esforço-recompensa, os CD, os TSB e os ASB do território pesquisado não estão em situação de sofrimento decorrente de estresse ocupacional, visto que 90,3\% ( $n=56$ ) da amostra está em estado de equilíbrio entre esforço despendido no trabalho e recompensa recebida.

Apesar de a pesquisa revelar resultados positivos e relevantes na questão da gestão do trabalho do município, os dados podem ter sofrido influência de alguns vieses, como, por exemplo, a população ser composta por profissionais mais velhos, 
experientes e com vínculo estável.

Destaca-se que, para além da razão entre esforço-recompensa, os resultados equilibrados em cada uma das três dimensões do questionário ERI revelaram uma quantidade considerável de participantes vivenciando situações de estresse ocupacional, o que os deixa propensos a desordens ao bem-estar no trabalho e na saúde.

Este tipo de pesquisa tem a sua importância no trabalho de sensibilização dos responsáveis pela gestão política e, especificamente, do trabalho no município. A partir dos expostos, espera-se que o estudo seja útil na reflexão e análise das condições de trabalho das ESB da ESF e na compreensão dos impactos do estresse sobre a vida e desempenho profissional, para que, assim, se efetivem práticas de vigilância e assistência à saúde dos trabalhadores e trabalhadoras de saúde do município.

\section{Referências}

Almeida, M. A. (2016). Análise da qualidade de vida e trabalho dos agentes comunitários de saúde de Sobral - Ceará. Dissertação (Mestrado em Saúde da Família) - Universidade Federal do Ceará, Sobral.

Altafim, C. A. C. (2018). Absenteísmo dos servidores de diferentes regimes de trabalho da Atenção Básica de Saúde em um município de médio porte. Dissertação (mestrado profissional) - Universidade Estadual de Campinas, Faculdade de Odontologia de Piracicaba, Piracicaba, SP.

Brasil. Ministério da Fazenda. Secretaria de Previdência. (2017). $1^{\circ}$ Boletim Quadrimestral sobre Benefícios por Incapacidade de 2017. Adoecimento Mental e Trabalho: a concessão de benefícios por incapacidade relacionados a transtornos mentais e comportamentais entre 2012 e 2016 . Brasília.

Cardoso, A. C., \& Morgado, L. (2019). Trabalho e saúde do trabalhador no contexto atual: ensinamentos da Enquete Europeia sobre Condições de Trabalho. Saúde e Sociedade, 28, 169-181.

Chor, D., Werneck, G. L., Faerstein, E., Alves, M. G. D. M., \& Rotenberg, L. (2008). The Brazilian version of the effort-reward imbalance questionnaire to assess job stress. Cadernos de Saúde Pública, 24:219-224.

Costa, D. G. S., \& Soares, N. (2018). Envelhecimento e velhices: heterogeneidade no tempo do capital. Serviço Social \& Realidade, 25(2).

de Brito, G. E. G., Mendes, A. D. C. G., dos Santos Neto, P. M., \& de Farias, D. N. (2016). Perfil dos trabalhadores da Estratégia Saúde da Família de uma capital do nordeste do Brasil. Revista de APS, 19(3).

de Oliveira A. M. N., \& de Araújo T. M. (2018). Situações de desequilíbrio entre esforço-recompensa e transtornos mentais comuns em trabalhadores da atenção básica de saúde. Trabalho, Educação e Saúde, 16(1):243-262.

do Prado, C. E. P. (2016). Estresse ocupacional: causas e consequências. Rev Bras Med Trab, 14(3), 285-9.

do Prado Tostes, M. F., da Silva, A. Q., Garçon, T. L.; Maran, E., \& Teston, E. F. (2017). Dualidade entre satisfação e sofrimento no trabalho da equipe de enfermagem em centro cirúrgico. Revista SOBECC, 22(1), 3-9.

dos Santos, J. D., Fillipiaki, C. K., Bermudes, D. F., Savaris, C. B., Rossa, L., \& Garrastazu, M. D. (2016). Suicídio dos profissionais e estudantes de odontologia associado às altas taxas de síndrome de Burnout - revisão de literatura. Ação Odonto, (1).

Hirschle, A. L. T., \& Gondim, S. M. G. (2020). Estresse e bem-estar no trabalho: uma revisão de literatura. Ciência \& Saúde Coletiva, $25,2721-2736$.

Iora, I. M. L. (2020). Alienação e exploração do trabalho em Karl Marx: atualidade e contribuições à sociologia contemporânea. Revista Contraponto, 7(3).

Lima, A. S., Farah, B. F., \& Bustamante-Teixeira, M. T. (2018). Análise da prevalência da síndrome de burnout em profissionais da Atenção Primária em Saúde. Trabalho, Educação e Saúde, 16(1), 283-304.

Maia, S. I. D. S. (2016). Suicídio (ou tentativa): acidente de trabalho ou doença de trabalho? Dissertação (mestrado). Universidade do Minho, Braga, Portugal.

Marcelino Filho, A., \& Araújo, T. M. D. (2015). Estresse ocupacional e saúde mental dos profissionais do centro de especialidades médicas de Aracaju. Trabalho, Educação e Saúde, 13:177-199.

Oliveira, I. V. P., de Braz, M. C. A., Urbano, S. C. F., dos Santos, I. C., \& Araki, A. T. (2018). Conhecimento de alunos e professores sobre medidas ergonômicas. Arquivos Em Odontologia, 54.

Schnall, P.L., Landsbergis, P. A., \& Baker, D. (1994). Job strain and cardiovascular disease. Annual Review of Public Health,15(1):381-411.

Siegrist, J., Starke, D., Chandola, T., Godin, I., Marmot, M., Niedhammer, I., \& Peter, R. (2004). The measurement of effort-reward imbalance at work: European comparisons. Social science \& medicine, 58(8):1483-1499.

Trindade, L. L., \& Lautert, L. (2010). Síndrome de Burnout entre os trabalhadores da Estratégia de Saúde da Família. Revista da Escola de Enfermagem da USP, 44(2), 274-279.

Zenkner, K. V., Denardin, E. F., de Jesus, A. A., Strom, B. R., da Silva, E. S., \& Carlesso, J. P. P. (2020). Saúde mental dos profissionais da saúde: o adoecimento de quem se dedica a cuidar a doença do outro. Research, Society and Development, 9(7), e916974747-e916974747. 